\title{
ASPECTOS ULTRA-SONOGRÁFICOS DA TROMBOSE DA VEIA PORTA*
}

\author{
Márcio Martins Machado ${ }^{1}$, Ana Cláudia Ferreira Rosa ${ }^{2}$, Orlando Milhomem da Mota ${ }^{3}$, Daniela \\ Medeiros Milhomem Cardoso ${ }^{4}$, Patrícia Medeiros Milhomem ${ }^{5}$, Leonardo Medeiros Milhomem ${ }^{5}$, \\ Rodrigo Alvarenga Nunes ${ }^{6}$, Thaís Bittencourt Gonçalves Teles ${ }^{7}$, Flávio Bittencourt Gonçalves \\ Teles $^{7}$, Letícia Martins Azeredo ${ }^{8}$, Nestor de Barros ${ }^{9}$, Giovanni Guido Cerri ${ }^{10}$
}

Resumo A trombose da veia porta pode estar associada a várias alterações, como a presença de tumores (por exemplo: hepatocarcinoma, doença metastática hepática e carcinoma do pâncreas), pancreatite, hepatite, septicemia, trauma, esplenectomia, derivações porto-cava, estados de hipercoagulabilidade (por exemplo: gravidez), em neonatos (por exemplo: onfalite e cateterização da veia umbilical) e desidratação aguda. Os autores discutem, neste artigo, os aspectos ultra-sonográficos da trombose de veia porta e alguns aspectos de relevância clínica.

Unitermos: Veia porta; Trombose de veia porta; Ultra-sonografia.

Abstract Ultrasonographic features of portal vein thrombosis.

Portal vein thrombosis may be associated with many alterations, such as the presence of tumors (for example, hepatocellular carcinoma, metastatic liver disease and carcinoma of the pancreas) as well as pancreatitis, hepatitis, septicemia, trauma, splenectomy, portacaval shunts, hypercoagulable conditions (for example, pregnancy), in neonates (for example, omphalitis and umbilical vein catheterization) and in acute dehydration. The authors discuss herein the sonographic features of portal vein thrombosis as well as some aspects of clinical relevance.

Keywords: Portal vein; Portal vein thrombosis; Ultrasound.

\section{INTRODUÇÃO}

A trombose da veia porta (Figura 1) é a causa mais comum de hipertensão portal pré-hepática. As fístulas arteriovenosas, envolvendo o fígado ou a circulação esplâncnica, são a segunda causa mais comum. Dentre as causas de trombose da veia porta, podemos ter a desidratação, estados

* Trabalho realizado no Departamento de Radiologia do Hospital das Clínicas da Faculdade de Medicina da Universidade de São Paulo, no Centro de Diagnóstico do Hospital Sírio Libanês, São Paulo, SP, e no Departamento de Doenças do Aparelho Digestivo do Hospital Araújo Jorge da Associação de Combate ao Câncer em Goiás, Goiânia, GO.

1. Professor Convidado do Departamento de Radiologia da Faculdade de Medicina da Universidade Federal de Goiás.

2. Médica Radiologista do Hospital das Clínicas da Faculdade de Medicina da Universidade Federal de Goiás.

3. Chefe do Departamento de Doenças do Aparelho Digestivo do Hospital Araújo Jorge da Associação de Combate ao Câncer em Goiás.

4. Residente do Departamento de Cirurgia da Faculdade de Medicina da Universidade Federal de Goiás.

5. Acadêmicos de Medicina da Faculdade de Medicina da Universidade de Ribeirão Preto.

6. Acadêmico de Medicina da Faculdade de Ciências Médicas da Universidade do Vale do Sapucaí.

7. Acadêmicos de Medicina da Faculdade de Medicina da Universidade Federal de Goiás.

8. Médica Ultra-sonografista do Departamento de Radiologia da Faculdade de Medicina da Universidade Federal de Minas Gerais.

9. Professor Doutor do Departamento de Radiologia da Faculdade de Medicina da Universidade de São Paulo. de choque, neoplasias malignas como hepatocarcinomas ou metástases hepáticas, carcinoma de pâncreas, leiomiossarcoma primário da veia porta, pancreatite crônica, hepatites, esplenectomia, estados de hipercoagulabilidade como a gravidez, pileflebite (processo infeccioso envolvendo a veia porta, decorrente, por exemplo, de apendicite aguda ou sepse abdominal), outras coagulopatias, invasão portal por tumores extra-hepáticos, compressão da veia porta (por pancreatite aguda, linfonodos), trauma, cateterismos, aumento da resistência ao fluxo (casos de cirrose, Budd-Chiari, esquistossomose hepatoesplênica), onfalite neonatal e mesmo após escleroterapia de varizes esofagianas ${ }^{(\mathbf{1})}$. As cirurgias de derivação (mesentérico-cava, porto-cava, espleno-renal distal) e de desconexão (desconexão ázigo-portal (DAPE) e esplenectomia) também podem apresen-

10. Professor Titular do Departamento de Radiologia da Faculdade de Medicina da Universidade de São Paulo.

Endereço para correspondência: Dr. Márcio Martins Machado. Rua Ruy Brasil Cavalcante, 496, Ed. Art-1, ap. 1001, Setor Oeste. Goiânia, GO, 74140-140. E-mail: marciommachado@ ibest.com.br

Recebido para publicação em 28/10/2004. Aceito, após revisão, em 7/1/2005. tar como complicação a trombose da veia porta ou de outros vasos relacionados ao tronco venoso espleno-mesentérico-portal.

Neste artigo, os autores discutem os aspectos de apresentação da trombose de veia porta, avaliando também aspectos clínicos relevantes neste contexto.

\section{DISCUSSÃO}

Embora a trombose completa de veia porta possa cursar com hipertensão portal, como referido anteriormente, tromboses parciais isoladas (Figura 2) não cursarão com hipertensão portal. Esses casos seriam reconhecidos nos exames realizados rotineiramente ou em pacientes apresentando fatores de risco associados. Identifica-se, nesses casos, a presença de material ecogênico aderido à parede do vaso, determinando obstrução parcial da luz. O Doppler pode ainda demonstrar fluxo portal com características razoavelmente preservadas. Nas tromboses totais, a ultra-sonografia mostra, usualmente, a veia porta com material ecogênico preenchendo a sua luz. Colaterais venosas portais, aumento do calibre da veia porta e transformação 


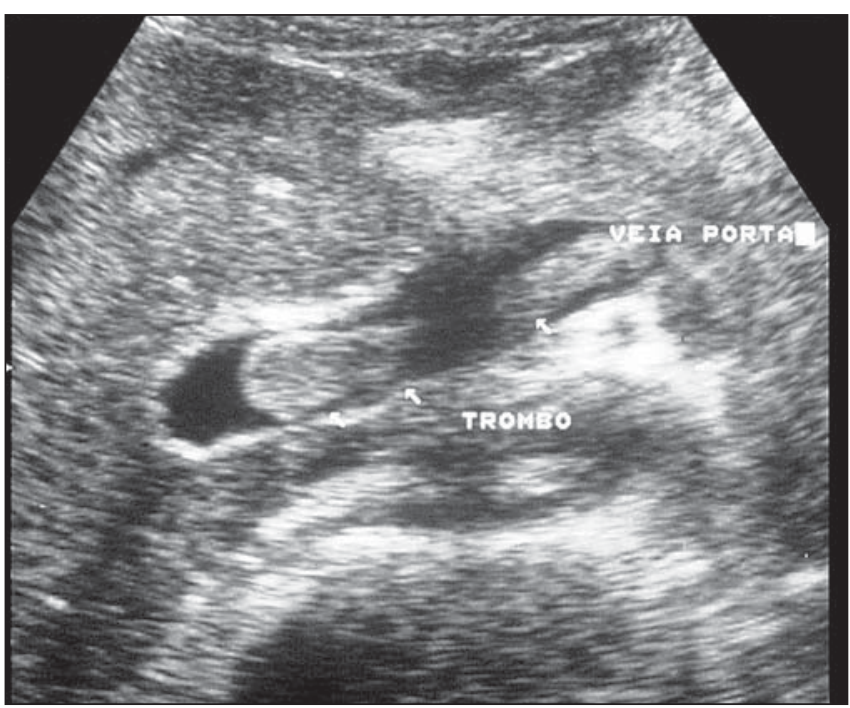

Figura 1. Trombose portal representada pela presença de material ecogênico no interior da veia porta.

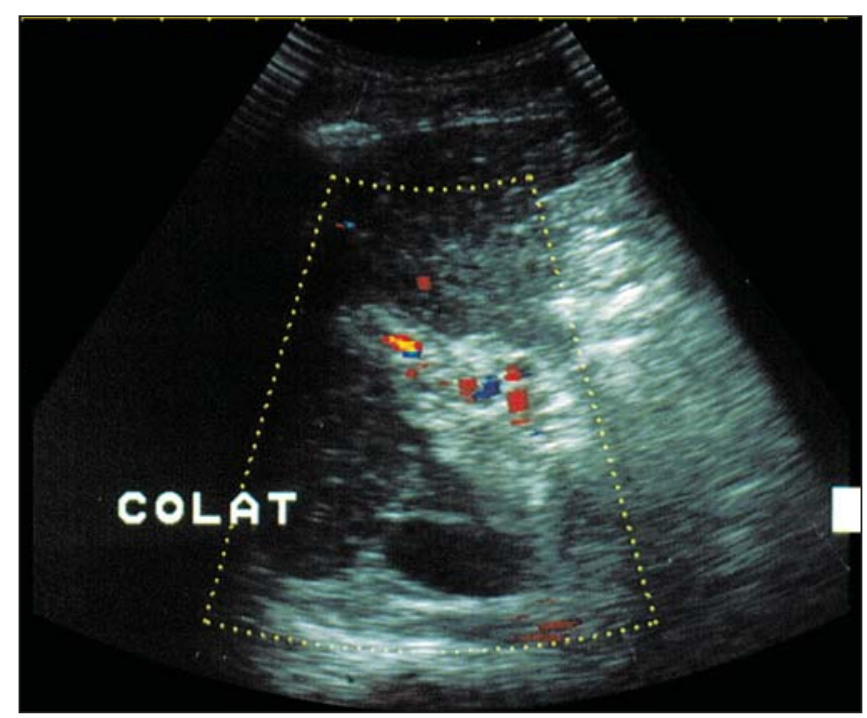

Figura 3. Trombose da veia porta. Doppler colorido mostrando a presença de fluxo pelas colaterais periportais (transformação cavernomatosa da veia porta).

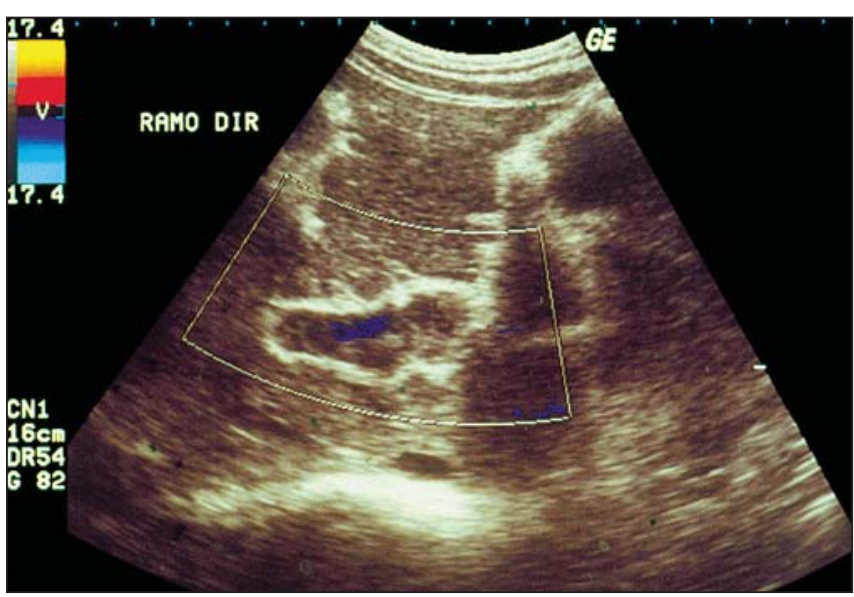

Figura 2. Trombose portal parcial. Doppler colorido demonstrando a presença de fluxo sanguíneo no lúmen residual da veia porta.

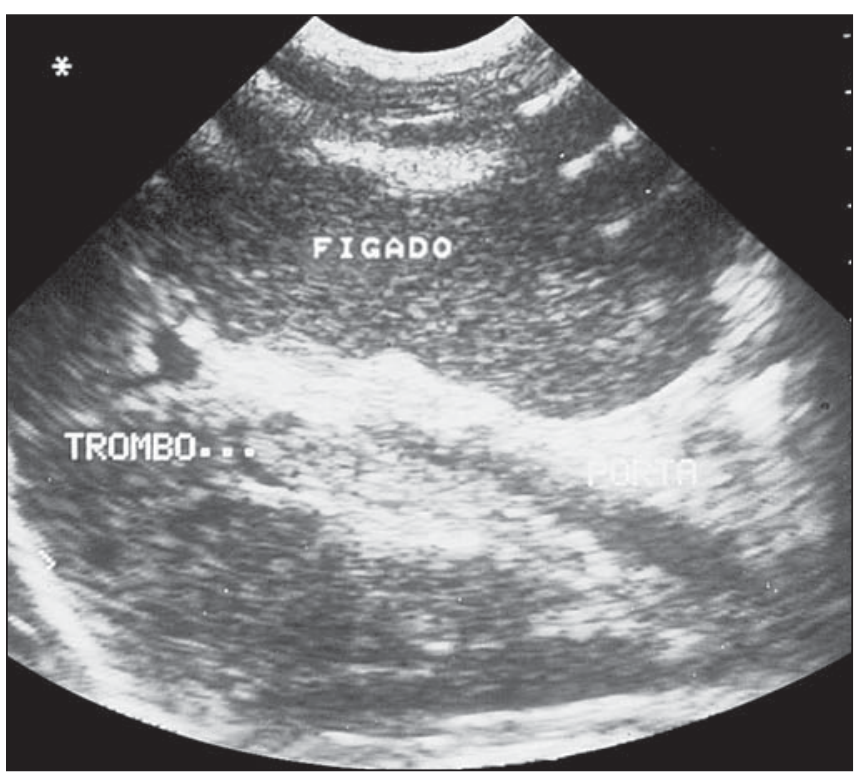

Figura 4. Trombose crônica da veia porta. Presença de hiperecogenicidade periportal (fibrose) e trombo hiperecogênico residual no interior da veia porta. cavernomatosa (Figura 3) também podem ser achados nos casos de trombose (especialmente nas tromboses totais).

Nos casos de tromboses mais recentes, ditas agudas, os trombos podem ser praticamente anecóides $^{(2)}$, dificultando o reconhecimento. Entretanto, o estudo dos vasos com aparelhos de alta resolução permite a identificação de material com alguma ecogenicidade (trombo) na maioria dos $\operatorname{casos}^{(\mathbf{3})}$. Mesmo nos casos de trombos agudos que não se apresentam ecogênicos, a utilização do Doppler colorido, espectral ou "power" Doppler (Doppler de amplitude) é essencial na demonstração da ausência de fluxo no vaso.

Nos casos de tromboses crônicas da veia porta (Figura 4), a ultra-sonografia demonstra, usualmente, veia porta de calibre reduzido e preenchida por material ecogênico.

Nos casos de tromboses completas (Figura 5) e na ausência de transformação cavernomatosa, o Doppler não identifica flu- xo. O Doppler pode detectar fluxo arterial na artéria hepática adjacente, devendo o examinador estar atento para esse fato. Outro aspecto que deve ser lembrado é que no caso de oclusões completas da veia porta podem se desenvolver as colaterais periportais.

Nesses casos de trombose crônica (especialmente as completas), podemos então encontrar a transformação cavernomatosa da veia porta (Figura 6), que consiste na presença de vasos tortuosos na topografia 


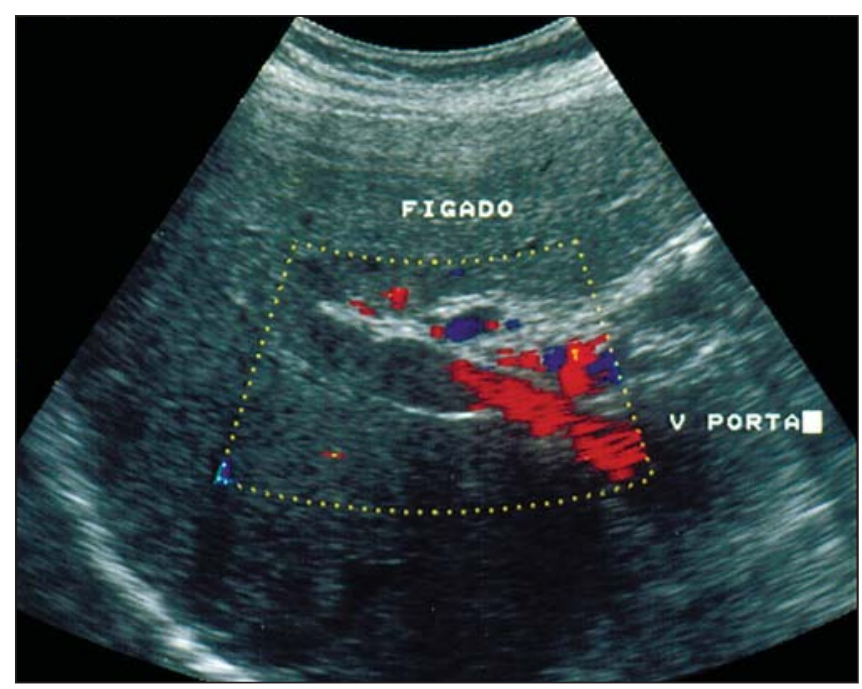

Figura 5. Trombose completa da veia porta.

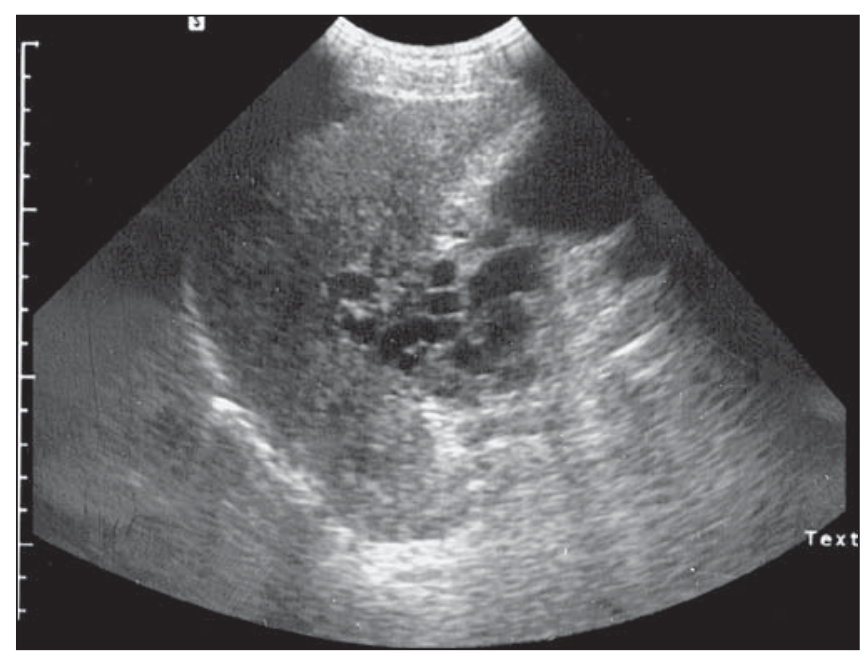

A

Figura 7. Trombose da veia porta com transformação cavernomatosa. A: Imagem em modo B demonstrando colaterais "serpiginosas" no hilo hepático. B: Doppler colorido demonstrando as colaterais.

da veia porta (na porta hepatis ou hilo hepático), que representam circulação colateral periportal (Figura 7). À ultra-sonografia, apresenta-se como estrutura ecogênica alongada, identificando-se inúmeros canais serpiginosos e tortuosos, representando os vasos colaterais ${ }^{(\mathbf{4})}$. Esses vasos serpiginosos podem representar a recanalização do próprio trombo, ou também colaterais perihilares (periportais) exuberantes, representando a circulação colateral extraluminar. Ao Doppler, notamos a presença desses canais tortuosos, apresentando fluxo (usualmente com velocidade reduzida) e com padrão comumente "achatado", sem as

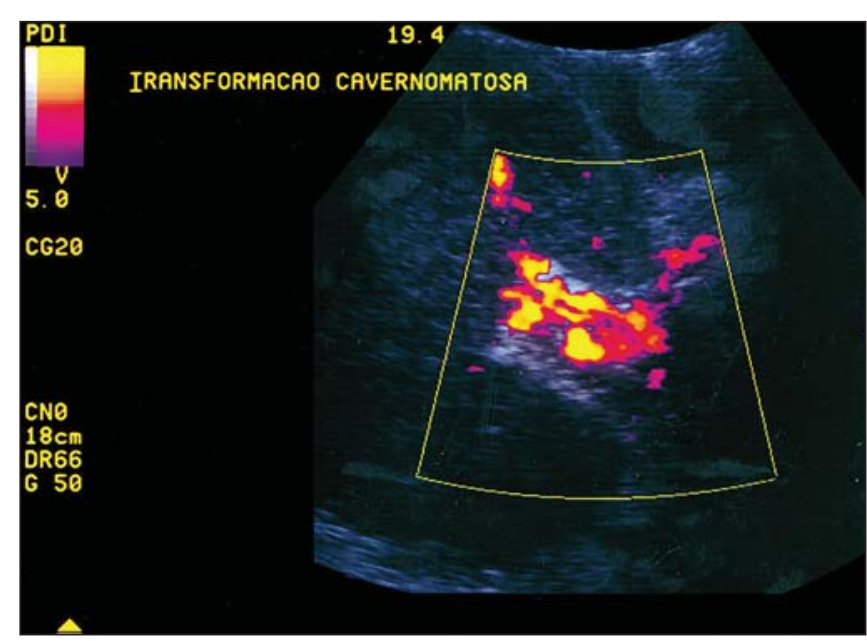

Figura 6. Doppler de amplitude demonstrando as colaterais exuberantes em caso de transformação cavernomatosa da veia porta.

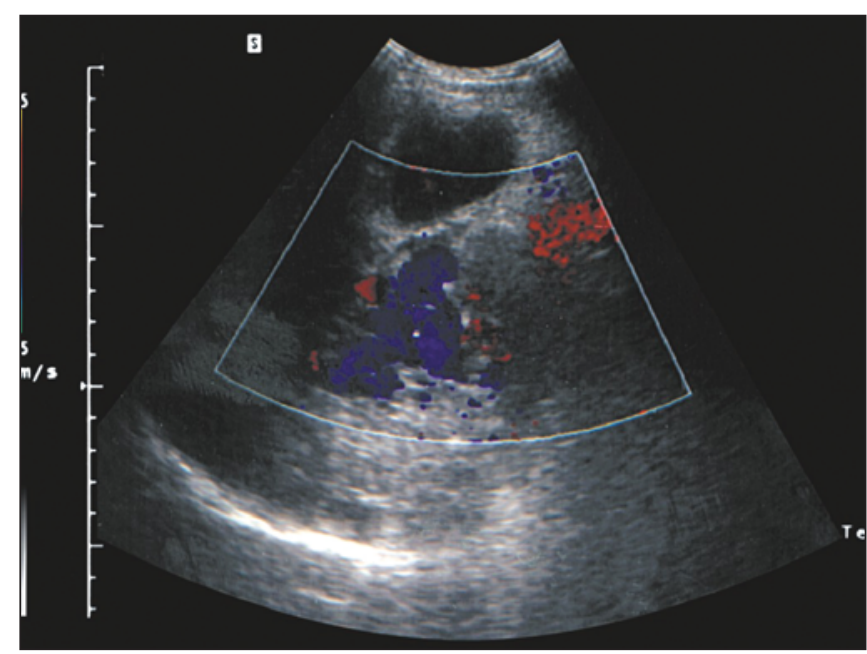

B alterações ondulatórias decorrentes do ciclo cardíaco, comumente observadas na veia porta normal ${ }^{(3)}$.

No estudo da veia porta, devemos estar atentos para o fato de que o fluxo pode não estar sendo visto, num determinado momento, devido a problemas técnicos, em vez da ausência real do fluxo. Podese tentar documentar a presença de fluxo em vasos adjacentes, com o mesmo aparelho e em profundidades semelhantes, para que se tenha segurança da ausência de fluxo na veia porta. De outro lado, a ausência do fluxo não indica, por si só, a presença de trombose oclusiva, podendo estar ocorrendo aumento da resistência hepática ao fluxo, indicando hipertensão portal importante ${ }^{(3)}$.

Um dos sinais indiretos de trombose da veia porta é o encontro de sinais de fluxo arterial de alta frequiência, que representam a tentativa de aumentar a circulação arterial hepática compensando a deficiência do fluxo venoso portal. Esse achado pode ser observado tanto no tronco da artéria hepática quanto em seus ramos intra-hepáticos ${ }^{(2)}$.

Todos esses aspectos descritos para a trombose da veia porta (tronco portal hilar) também se aplicam às tromboses de 
seus ramos direito, esquerdo e ramos menores intra-hepáticos, sendo o aspecto sonográfico e ao Doppler semelhante àquele encontrado no tronco da veia porta.

A trombose dos ramos intra-hepáticos da veia porta pode ser devida à extensão do processo a partir do tronco da veia porta (mais comumente), ou ocorrer isoladamente, sem trombose portal associada. Deve ser enfatizado, também, que o processo de trombose portal pode se estender ou se originar de outros vasos do tronco venoso espleno-mesentérico-portal, além daquele representado pelo tronco da veia porta. Identifica-se material ecogênico ocluindo total ou parcialmente a luz do vaso. Ao Doppler, observa-se ausência de fluxo (nos casos de tromboses oclusivas) ou fluxo periférico (trombose parcial). Principal- mente ao nível dos ramos portais intrahepáticos (ramo direito, esquerdo, ou ramos mais periféricos), a luz pode não ser identificada, observando-se apenas bandas ecogênicas, representando os trombos. Em alguns desses casos, o Doppler pulsado pode revelar a presença de algum fluxo residual, mesmo na ausência de lúmen vascular identificável $^{(3)}$.

Na oclusão portal, podem ser identificadas colaterais venosas exuberantes, sendo que as colaterais pericolecísticas (Figura 8) são particularmente evidentes nesses casos ${ }^{(\mathbf{5})}$. Nessa situação, elas representam a via colateral do sangue da circulação esplâncnica em direção ao fígado (colaterais hepatopetais). Embora referidas por alguns como quase patognomônicas da presença associada de trombose da veia porta $^{(3)}$, outros autores têm observado esse achado em pacientes com hipertensão portal cirrótica ou esquistossomótica, sem trombose portal associada ${ }^{(4,6)}$.

Estudos com grande número de pacientes com hipertensão portal cirrótica indicam que a ocorrência de trombose portal parcial pode ser verificada em $1,8 \%$ dos casos, e a total, em $4,4 \%{ }^{(7)}$. Ainda com relação à trombose da veia porta (ou de seus ramos), é freqüente a preocupação com essa avaliação nos pacientes portadores de neoplasias hepáticas primárias ou secundárias. Nesse sentido, embora existam variações, alguns autores relataram a presença de oclusão da veia porta em aproximadamente $25 \%$ dos pacientes com carcinoma hepatocelular e em $1 \%$ dos pacientes com metástases $^{(4)}$.

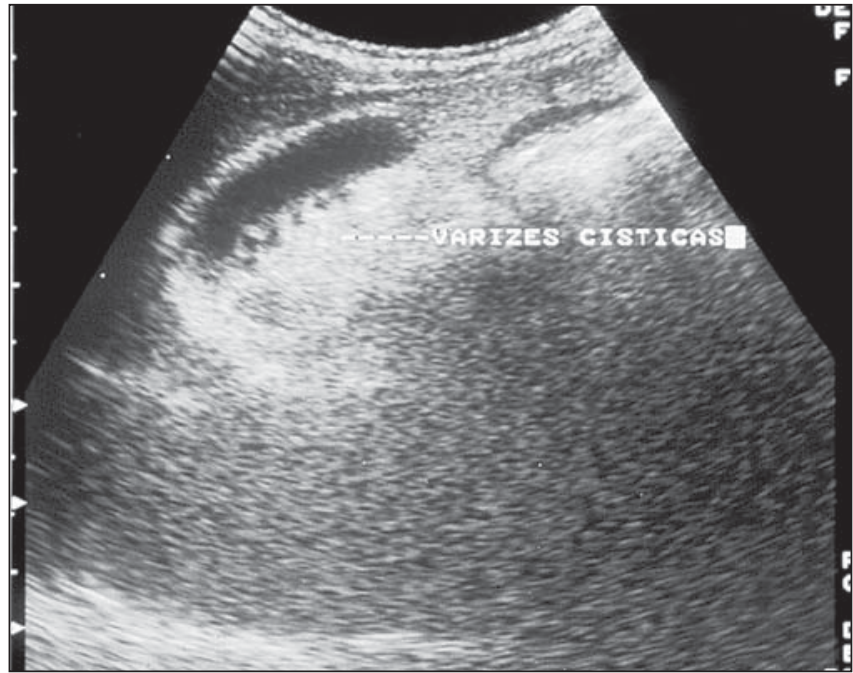

A

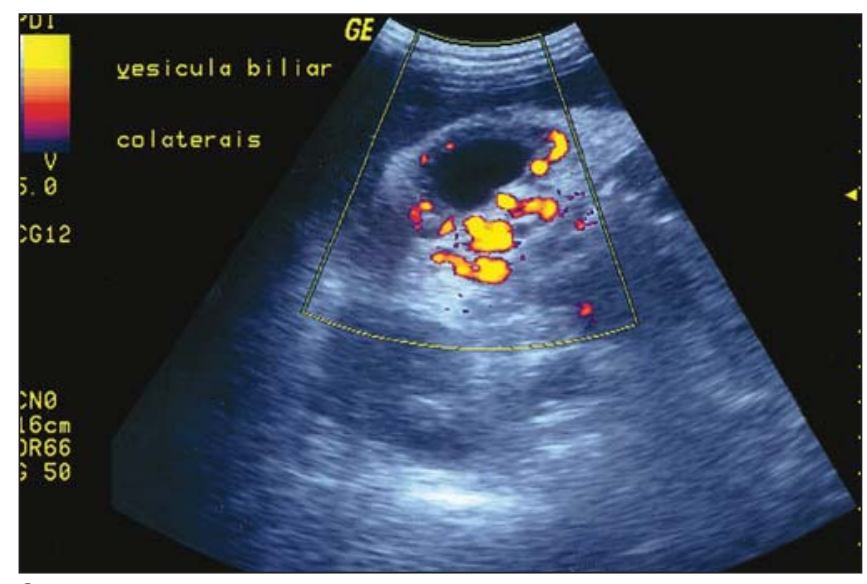

C

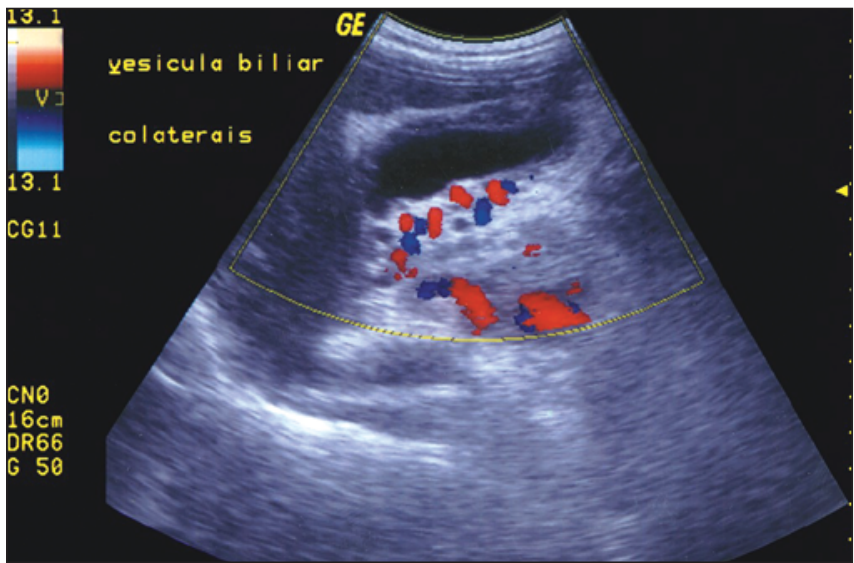

B

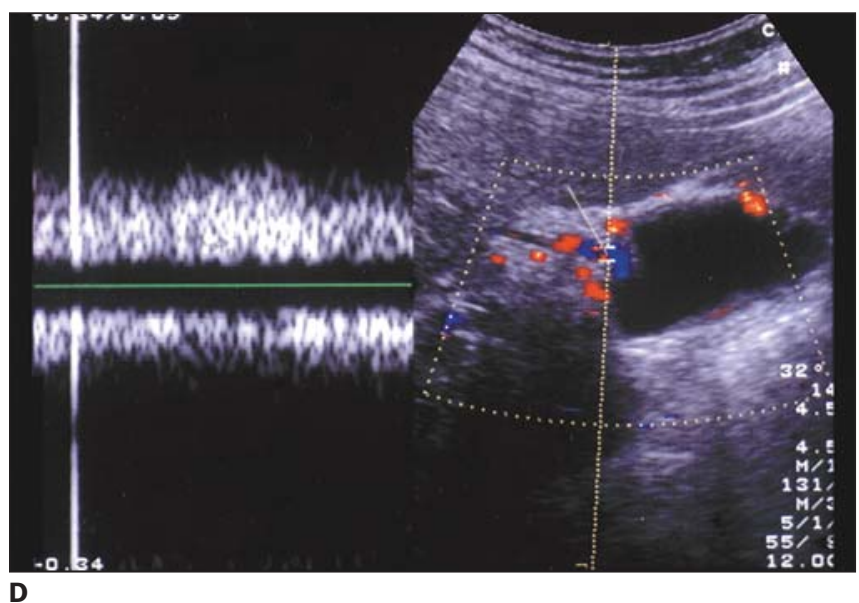

Figura 8. Colaterais pericolecísticas em paciente com trombose da veia porta. A: Imagem em modo B. B: Imagem com Doppler colorido. C: Imagem com Doppler de amplitude. D: Imagem com Doppler espectral, demonstrando fluxo venoso. 
O desenvolvimento de fluxo hepatofugal é outra alteração que pode ocorrer na hipertensão portal, sendo que estudos de angiografia indicam a presença dessa alteração, variando de $0 \%$ a $14,8 \%$ dos casos avaliados $^{\left({ }^{(8-11)}\right.}$. Muitos trabalhos têm indicado índices intermediários de fluxo hepatofugal, oscilando de $5,3 \%$ a $8,3 \%{ }^{(\mathbf{2 , 1 2})}$.

A reversão do fluxo indica a presença de alta resistência ao fluxo portal intra-hepático. Nessas situações, ocorre o desenvolvimento de amplos vasos colaterais (colaterais porto-sistêmicas), para dar escoamento alternativo ao fluxo sanguíneo, que não está conseguindo atravessar o leito hepático em direção à circulação sistêmica. Nesse momento, a veia porta pode reduzir o seu calibre, mesmo na vigência de hipertensão portal. O sangue estará sendo desviado para setores de menor resistência (sistêmico), pelas colaterais, "descomprimindo" a veia porta (sistema portal hipertenso) ${ }^{(2)}$. Também foi demonstrado que na vigência da reversão do fluxo sanguíneo (fluxo hepatofugal) diminui-se o risco de hemorragias digestivas, estando comumente o diâmetro da veia porta reduzido ${ }^{(2)}$.

Outra correlação que existe é entre a presença de fluxo hepatofugal na veia es- plênica e o desenvolvimento de encefalopatia. Isto ocorreria devido ao desvio do fluxo portal para a circulação sistêmica através das colaterais ao nível do hilo esplênico e, principalmente, através de anastomoses espleno-renais espontâneas ${ }^{(\mathbf{1 3})}$.

\section{CONCLUSÃO}

Os autores concluem que as causas de trombose da veia porta são multifatoriais, e que devemos estar sempre atentos para esta possibilidade, entendendo que a ultrasonografia com Doppler constitui-se em método não invasivo de grande utilidade na sua identificação.

\section{REFERÊNCIAS}

1. Cerri GG, Mólnar LJ, Paranaguá-Vezozzo DC. Doppler. São Paulo: Sarvier, 1996;124-135.

2. Bolondi L, Gaiani S, Barbara L. Liver and portal hypertension. In: Taylor KJW, Burns PN, Wells PNT, editors. Clinical applications of Doppler ultrasound. 2nd ed. Philadelphia: Lippincott-Raven, 1995;133-154.

3. Weill FS. Ultrasound diagnosis of digestive diseases. 4th ed. Berlin: Springer-Verlag, 1996;121135.

4. Cosgrove DO. Ultrasound contrast agents. In: Meire H, Cosgrove D, Dewbury K, Farrant P, editors. Abdominal and general ultrasound. London: Churchill Livingstone, 2001;69-79.

5. Cerri GG, Habr-Gama A, Paranaguá-Vezozzo D,
Machado MCC, Magalhães A, Pinotti HW. Doppler demonstration of cystic vein dilatation secondary to portal vein thrombosis. Surg Endosc 1991;5:9293.

6. Machado MM, Rosa ACF, Cerri GG. Doenças hepáticas difusas, hipertensão portal e transplante de fígado. In: Cerri GG, Oliveira IRS, editores. Ultrasonografia abdominal. Rio de Janeiro: Revinter, 2002;55-124.

7. Gaiani S, Bolondi L, Fenyves D, Zironi G, Rigamonti A, Barbara L. Effect of propranolol on portosystemic collateral circulation in patients with cirrhosis. Hepatology 1991;14:824-829.

8. Foster DN, Herlinger H, Miloszewski KJA, et al. Hepatofugal portal blood flow in hepatic cirrhosis. Ann Surg 1978;187:179-182.

9. Okazaki K, Miyazaki M, Onishi S, Ito K. Effects of food intake and various extrinsic hormones on portal blood flow in patients with liver cirrhosis demonstrated by pulsed Doppler with Octoson. Scand J Gastroenterol 1986;21:1029-1038.

10. Rector WG Jr, Hoefs JC, Hossack KF, Everson GT. Hepatofugal portal flow in cirrhosis: observations on hepatic hemodynamics and the nature of arterioportal communications. Hepatology 1988;8:1620.

11. Burcharth F, Aagaard J. Total hepatofugal portal blood flow in cirrhosis demonstrated by transhepatic portography. ROFO 1988;148:47-49.

12. Kawasaki T, Moriyasu F, Nishida O, et al. Analysis of hepatofugal flow in portal venous system using ultrasonic Doppler duplex system. Am J Gastroenterol 1989;84:937-941.

13. Ohnishi K, Saito M, Nakayama T, et al. Portal venous hemodynamics in chronic liver disease: effect of posture change and exercise. Radiology 1985;155:757-761. 